\title{
Using Narrow Line-Width Laser to Measure the Thickness and Refractive Index of the Film
}

\section{Junjie Fang}

Shanghai Pinghe School, Shanghai, China

Correspondence to: Junjie Fang, fangjunjie@shphschool.com

Keywords: Particle Swamp Optimization, Narrow-Lined Laser, Thin-Film Thickness and Refractive Index

Received: September 28, $2020 \quad$ Accepted: November 21, $2020 \quad$ Published: November 24, 2020

Copyright (c) 2020 by author(s) and Scientific Research Publishing Inc.

This work is licensed under the Creative Commons Attribution International License (CC BY 4.0).

http://creativecommons.org/licenses/by/4.0/

\section{(c) (1) Open Access}

\section{ABSTRACT}

We demonstrate applications of a novel setup which is used for measuring the relative phase difference between $S$ and $P$ polarization at an oblique incidence point in optically denser medium by analyzing the relative frequency shift of adjacent axial modes of $S$ and $P$ resonances of a monolithic folded Fabry-Perot cavity (MFC). The relative phase difference at a reflection point $A$ in an optically denser medium is inferred to be around $-167.4^{\circ}$ for a confocal cavity and $-201.1^{\circ}$ for a parallel cavity. Given the $n_{1}, n_{3}, \varphi_{1}, \varphi_{3}, \lambda$, and $\Delta$, the elliptic formula $\tan (\psi) \exp (i \Delta)=R_{p} / R_{s}$ is used to find a solution for thickness $\mathrm{d}$ and refractive index $n_{2}$ of the thin film coated on point $\mathrm{A}$, where $R_{s}$ and $R_{p}$ are total refractive index of $s$ and $p$ component of light related to two unknown values. Since it is hard to deduce an analytical solution for thickness and refractive index of the film, we firstly used exhaustion method to find the set of solution about thickness and refractive index when assumed there is no light absorption by the film and then Particle Swarm Optimization (PSO) to find a set of solution of thickness and complex refractive index which accounts the light absorption by the film.

\section{INTRODUCTION}

Diode lasers have been widely adopted due to their ease of operation, low cost, and wide wavelength coverage in the visible and near-infrared spectrum. A free-running laser diode typically has a very broad emission profile. Such linewidths can be undesirable for physics experiments involving diode lasers, but thankfully, techniques have been developed that can significantly narrow the emission profile of these lasers. The most common technique utilizes an external diffraction grating in the "Extended Cavity Diode Laser" (ECDL) configuration, in which a diffraction grating sends a narrow part of the broad emitted spectrum back into the diode, thus creating feedback. This can typically narrow the linewidth of the laser to a few megahertz or less. 
The narrow line-width laser has a number of desired properties. For instance, even there is small change in direction of polarization of phase, the change can be detected since the reflected laser cannot reach the resonance with the original narrow line-width laser. Using this property, having the inspiration from mechanism of ellipsometry, we developed a more advanced method to measure the thickness and refractive index of a thin film.

Ellipsometry is a versatile and powerful optical technique for the investigation of the complex refractive index or dielectric parameters and is related to sample properties.

When a linearly polarized light is incident on a film, due to difference in light absorption and phase change to $s$ and $p$ polarized light, the light after reflection will become an elliptically polarized light. The fundamental method of Ellipsometry is to detect the change in relative amplitude and phase difference of $s$ and $\mathrm{p}$ polarization of incident wave after being reflected from a surface coated with film. These changes can be described by ellipsometry parameter $\psi$ and $\Delta$. The equation between $(\psi, \Delta)$ and $(d, n)$, the thickness and refractive index, can be determined. Theoretically, analytical solutions of thickness and refractive index of the film can be derived. Typically, ellipsometry is applied only when light travels from air to an optically denser medium. However, for the case of light travelling from an optically denser medium to an optically thinner medium, it does not work. In this letter, we demonstrate a novel setup which is used for measuring the relative phase difference between $\mathrm{S}$ and $\mathrm{P}$ at an oblique incidence point in an optically denser medium by analyzing the relative frequency shift of adjacent axial modes of $\mathrm{S}$ and $\mathrm{P}$ resonances of a monolithic folded Fabry-Perot cavity (MFC). Our work firstly uses newly designed system to attain the necessary parameter for measuring the thickness and refractive index of the film, and uses the same method of data processing in ellipsometry to derive a solution of thickness and refractive index of a film. According to the hardness to attain an analytic solution, particle swamp optimization (PSO) algorithm and exhaustion method will be introduced to solve the problem.

The research of Christopher Luetjen, Jonathan Hallsted, and Michaela Kleinert in 2012 had focused on the similar topic of our study, but their method does not count the change in direction of polarization, and requires the thickness of film to be known in order to calculate the refractive index, while our method can calculate the refractive index without knowing thickness. Compared to previous study, the combination can be more effective in solving the problem of measuring.

\section{PRINCIPLE AND EXPERIMENT}

In our experiments, the first medium is optically dense than the second; $n^{\prime}=0.697$ is the relative index of refraction. In the case of the reflected wave, the phase changes of each component of the reflected wave will depend on incidence magnitudes. By applying Fresenel formula, which are shown by Equation (1) and (2), Equation (3) and (4) can be derived.

$$
\begin{gathered}
r_{s}=\cos \theta-i \sqrt{\sin ^{2} \theta-n^{\prime 2}} / \cos \theta+i \sqrt{\sin ^{2} \theta-n^{\prime 2}}=\left|r_{s}\right| \mathrm{e}^{i \delta_{s}} \\
r_{p}=n^{\prime 2} \cos \theta-i \sqrt{\sin ^{2} \theta-n^{\prime 2}} / n^{\prime 2} \cos \theta+i \sqrt{\sin ^{2} \theta-n^{\prime 2}}=\left|r_{p}\right| \mathrm{e}^{i \delta_{p}} \\
\tan \left(\delta_{s} / 2\right)=-\sqrt{\sin ^{2} \theta-n^{\prime 2}} / \cos \theta \\
\tan \left(\delta_{p} / 2\right)=-\sqrt{\sin ^{2} \theta-n^{\prime 2}} /\left(n^{\prime 2} \cos \theta\right)
\end{gathered}
$$

$r_{s}$ and $r_{p}$ represent the refractive index of $s$ and $p$ polarization and $\delta_{s}$ and $\delta_{p}$ represent the phase change

$$
\Delta_{2}=\delta_{s}-\delta_{p}=2 \tan ^{-1}\left(\cos \theta \sqrt{\sin ^{2} \theta-n^{\prime 2}} / \sin ^{2} \theta\right)
$$

The separated result for $\delta_{p}$ and $\delta_{p}$ are both shown in Figure 1. The result of Equation (5) is illustrated in Figure 2. 


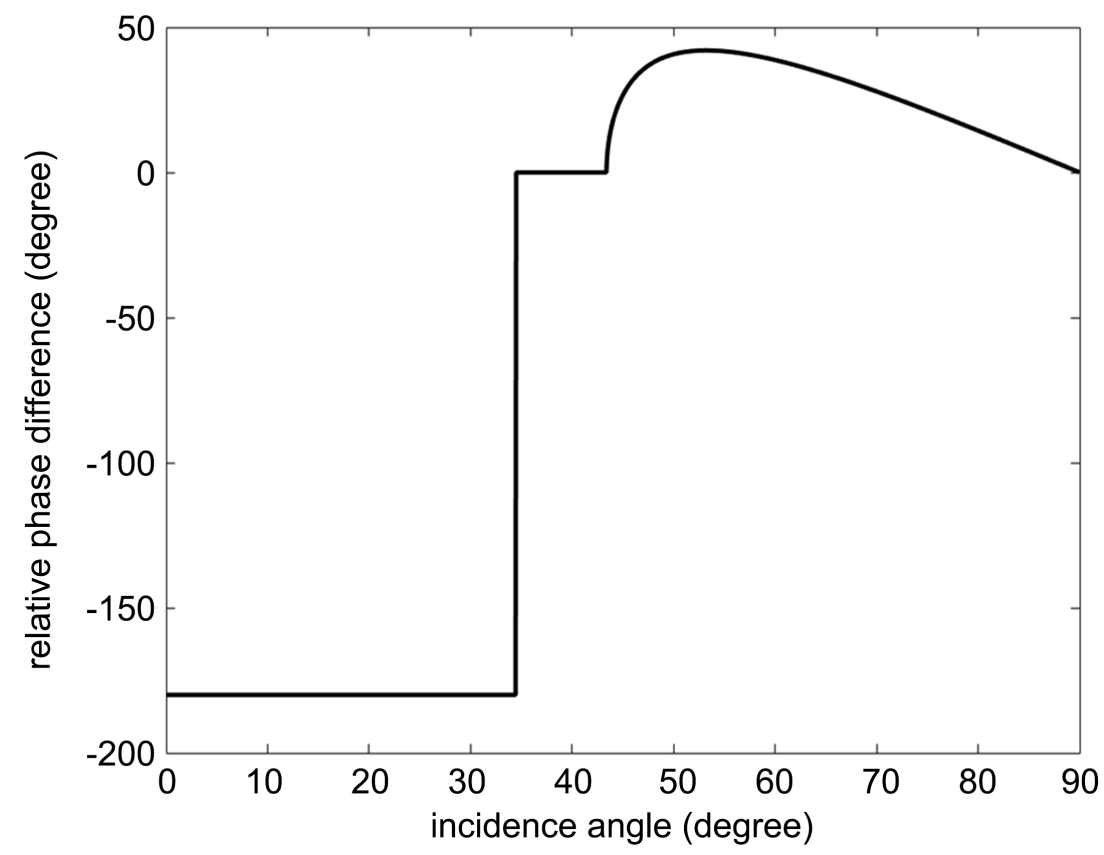

Figure 1. The phase difference of $S$ polarization (red) and P polarization (blue) versus incidence angle.

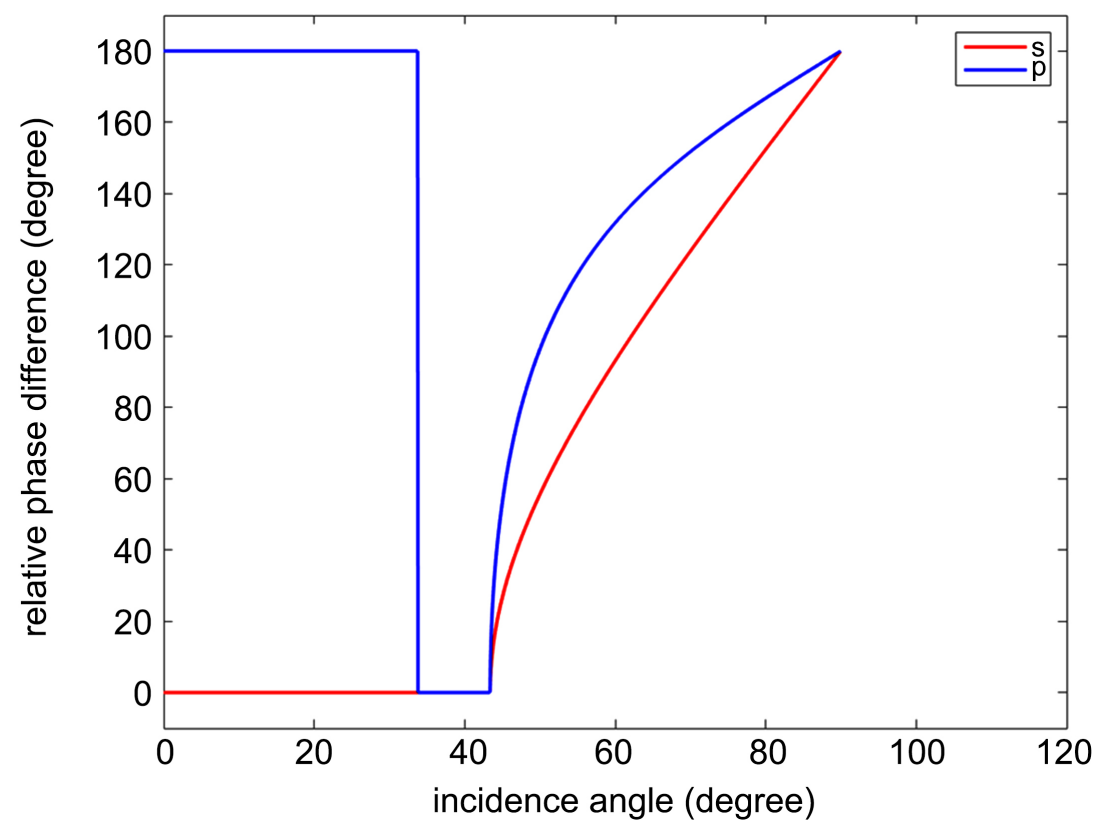

Figure 2. The relative phase difference between $\mathrm{S}$ and $\mathrm{P}$ versus incidence angle.

The measurement setup is shown in Figure 3. The laser diode is operating at the wavelength of 690 $\mathrm{nm}$. The temperature of the laser diode is stabilized at room temperature by a thermoelectric cooler. The laser beam is incident on a diffraction grating with 2400 grooves $\mathrm{mm}^{-1}$ with a spectral resolution of 50 $\mathrm{GHz}$. The S and P resonances are separately acquired by adjusting the half-wave plate, which is used for changing the polarization direction of incidence. While a resonance signal appears when the laser frequency is equal to the resonant frequency of the MFC by controlling the PZT, the feedback laser's polari- 
zation direction will be rotated by the magnetic field and reflected by the PBS into the PD detector. The signal will be recorded as a voltage pulse.

Figure 4 shows the procedure of changing the $\mathrm{S}$ (green) and $\mathrm{P}$ (blue) components of the resonance of a confocal MFC. In this figure, $3.413 \mathrm{GHz}$ of free spectral range is obtained by the scanning laser frequen$\mathrm{cy}$, in which the higher order mode appears in the middle of the spectral range because of incident pattern mismatching to the confocal MFC. The phase difference of $\mathrm{s}$ and $\mathrm{p}$ component of the incidence light is determined by:

$$
\Delta=2 \pi \Delta f t
$$

In Equation (6), $t$ is referring to the time lag caused by the reflection in the MFC, which is equal to $t=n 2 l / c . n$ is the refractive index of the MFC, 1 is focal length. According to Figure 4, for adjacent axial modes of the Sand $\mathrm{P}$ resonances of the MFC, $\Delta f_{0}$ is a $1.030 \mathrm{GHz}$ spectral shift, therefore the frequency difference between $S$ and $\mathrm{P}$ polarization is determined by $\Delta f=\mathrm{NFSR} \pm \Delta f_{0}$, in which $\mathrm{N}$ represents the number of FSRs the $\Delta f$ has, and FSR represents the free spectral range with a value of $3.413 \mathrm{GHz}$ for the case of a confocal MFC. We roughly estimate $\Delta f=\mathrm{NFSR}+\Delta f_{0}$ and $\Delta f=\mathrm{NFSR}-\Delta f_{0}[1]$ respectively, and determine which expression is proper to ensure that expressions (6) and (7) are the same quantity for integer $\mathrm{N}$ and the theoretical value of the relative phase difference at the reflection point A, shown in Figure 5. From Equation (7), we can get the value of the total relative phase difference, 1 . On the other hand, the total relative phase difference is expressed by

$$
\Delta=2 \Delta_{1}+2 \Delta_{2}+\Delta_{3}+\Delta_{4}
$$

where $\Delta_{1}, \Delta_{2}, \Delta_{3}$ and $\Delta_{4}$ are the relative phase differences between $\mathrm{S}$ and $\mathrm{P}$ resonances caused by points $\mathrm{A}$, $\mathrm{B}, \mathrm{C}$ and $\mathrm{D}$ respectively. For the total internal reflection point $\mathrm{B}$, a value of $43.49^{\circ}$ is calculated for $\Delta_{2}$ by Equation (7). At the normal incidence points $C$ and $D$, a relative phase difference of around $\Delta_{3} \approx \Delta_{4} \approx$ $-180^{\circ}$ is obtained. According to equation (5), therefore, the relative phase difference at the reflection point A, 11 , is inferred to be around $-164.7^{\circ}$, which has some deviation from $-180^{\circ}$. The deviation, we think, is caused by the coating films of plane S1, shown in Figure 5 .

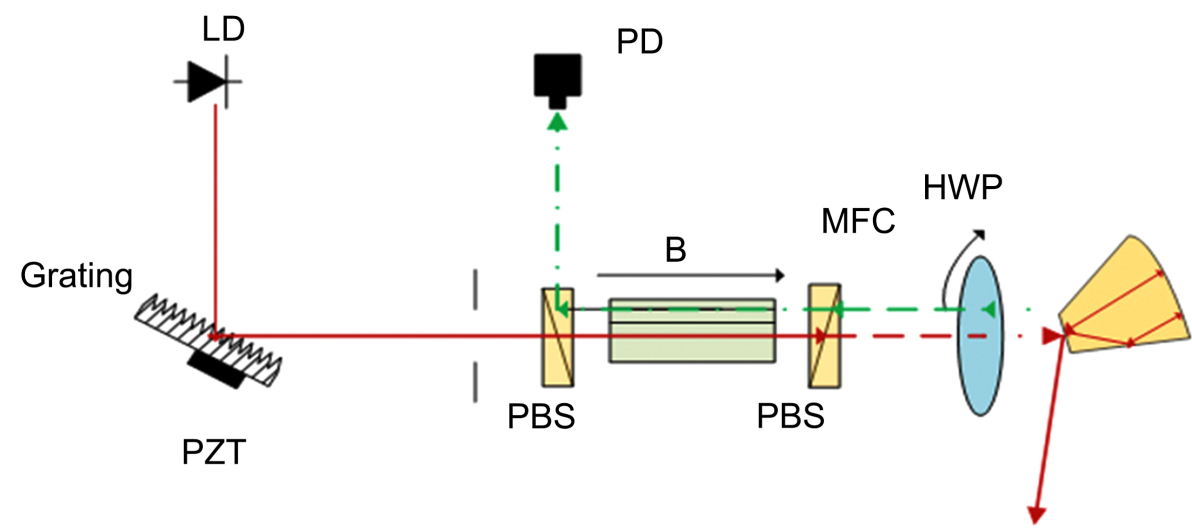

Figure 3. The setup for obtaining the relative phase difference inside theoretically denser medium of the MFC: LD, narrow line-width laser; HWP; half-wave plate; PZT, piezoelectric transducer; MFC, monolithic folded FP confocal cavity; PD, photodetector; PBS polarizing beam splitter; red arrow, direction of incident light; green arrow, resonant feedback; solid line, original polarization direction; dotted line, $45 \mathrm{de}-$ gree from original polarization; dashed line, 45 degree from original polarization; dash dotted line 90 degree from original polarization. After reflection of $s$ and $p$ polarization. 


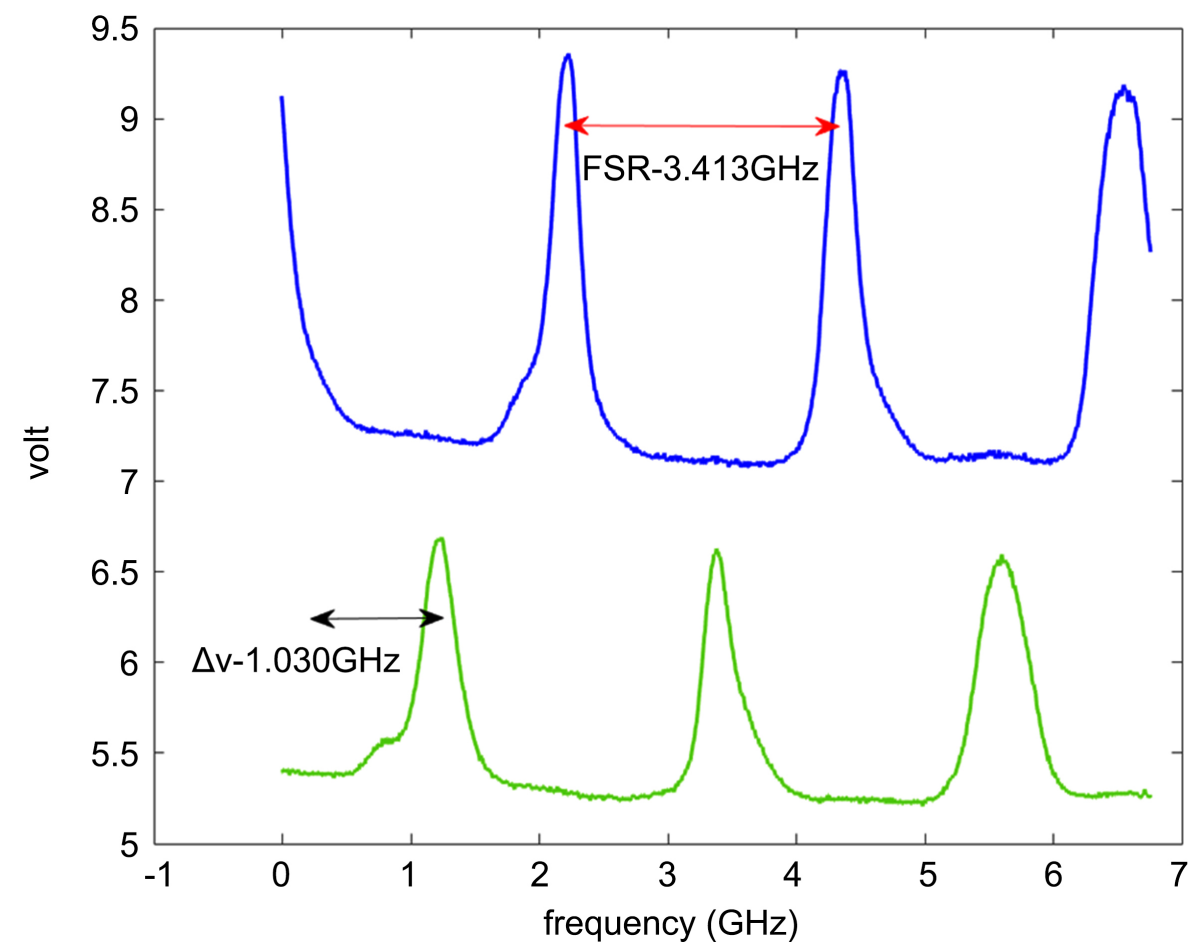

Figure 4. Relative phase difference between $S$ and $P$ versus incidence angle. The red points marked $A, B, C, D$ represent the corresponding resonant reflection points inside the MFC respectively.

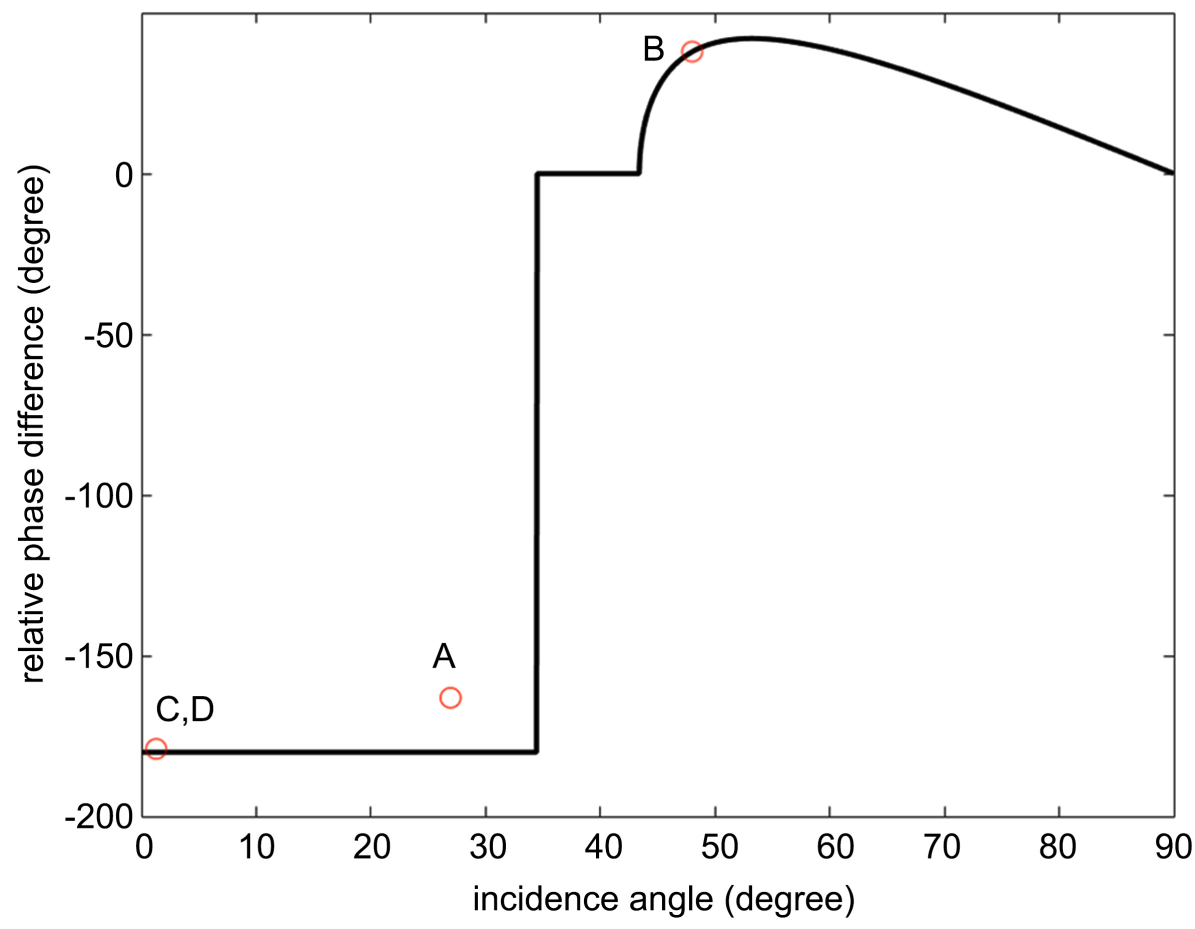

Figure 5. Relative phase difference between $S$ and $P$ versus incidence angle. The red points marked $A, B, C, D$ represent the corresponding resonant reflection points inside the MFC respectively. 


\section{REFRACTIVE INDEX AND THICKNESS}

The refractive index and thickness are derived based on the graphical illustration of light route in Figure 6.

For finding the thickness and refractive index of the film, we apply the same method as ellipsometry

$$
\begin{gathered}
r_{1 p}=\tan \left(\varphi_{1}-\varphi_{2}\right) / \tan \left(\varphi_{1}+\varphi_{2}\right) \\
r_{1 s}=\sin \left(\varphi_{1}-\varphi_{2}\right) / \sin \left(\varphi_{1}+\varphi_{2}\right)
\end{gathered}
$$

In the interface $n_{2}-n_{3}$, we have the refractive rate of

$$
\begin{aligned}
& r_{2 p}=\left(n_{3} \cos \varphi_{2}-n_{2} \cos \varphi_{3}\right) /\left(n_{3} \cos \varphi_{2}+n_{2} \cos \varphi_{3}\right) \\
& r_{2 s}=\left(n_{2} \cos \varphi_{2}-n_{3} \cos \varphi_{3}\right) /\left(n_{2} \cos \varphi_{2}+n_{2} \cos \varphi_{3}\right)
\end{aligned}
$$

From the diagram, we can tell the light experience a number of interferences. By using the equation for interference, we can determine the total refractive index.

$$
\begin{array}{r}
R_{p}=\left(r_{1 p}+r_{2 p} \exp (-2 i \delta)\right) /\left(1+r_{1 p} r_{2 p} \exp (-2 i \delta)\right) \\
R_{s}=\left(r_{1 s}+r_{2 p} \exp (-2 i \delta)\right) /\left(1+r_{1 s} r_{2 s} \exp (-2 i \delta)\right)
\end{array}
$$

$\delta$ is the phase difference caused by a single reflection at the $n_{2}-n_{3}$ boundary caused by the thickness of the film, which can be calculated by equation $\delta=2 \pi\left(\frac{d_{1}}{\lambda}\right) n_{2} \varphi_{1}$.

We can use the elliptical parameter $\psi$ and $\Delta$ to describe the ratio between total refractive index by using ellipsometry equation:

$$
\tan (\psi) \exp (i \Delta)=\frac{R_{p}}{R_{s}}
$$

The original amplitude of $\mathrm{s}$ and $\mathrm{p}$ component after passing the HWP is same. As the result, we can have Equation (7). The $\left(E_{p f} / E_{s f}\right)$ is the amplitude ratio between $p$ and $s$ direction component after the reflection from the previous surface, which is the measured value. $\Delta_{1}$ is already determined. The difference between theoretical phase difference and the actually $\Delta_{1}$ calculated is equal to the phase change caused by the coated film

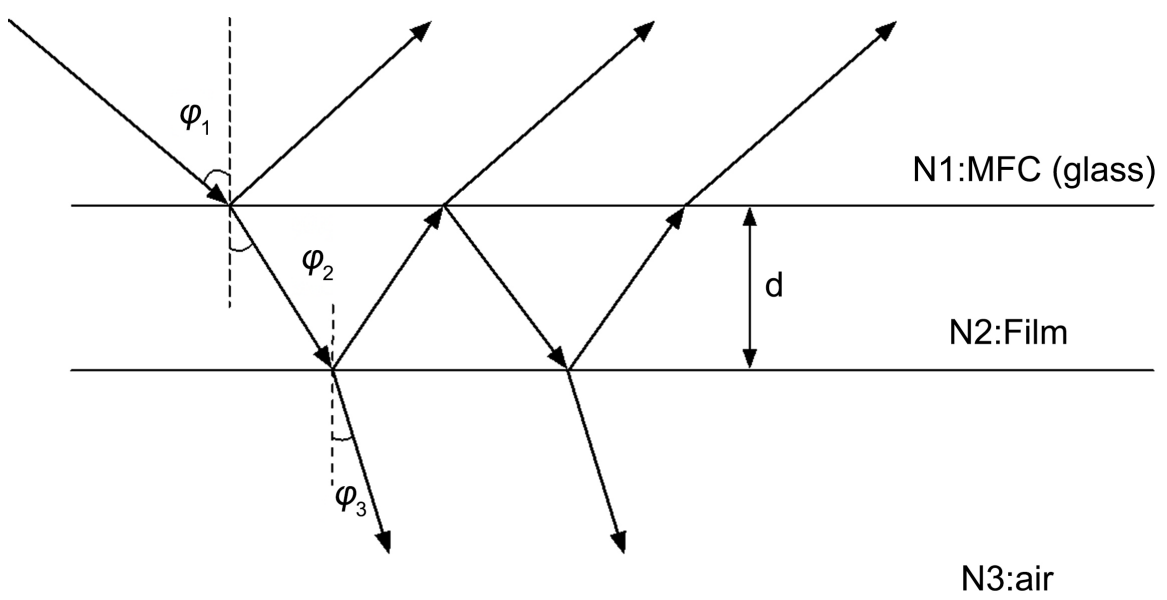

Figure 6. Graphical illustration to the light route of oblique incident laser at B point of MFC. 


$$
\left\{\begin{array}{l}
\tan (\psi)=\frac{E_{p f}}{E_{s f}} \\
\Delta=\Delta_{1}+\pi
\end{array}\right.
$$

$\left(R_{p} / R_{s}\right)$ can be written in an expression containing $\left(n_{1}, n_{2}, n_{3}, \varphi_{1}, \varphi_{2}, \varphi_{3}, d, \lambda, \theta\right)$, where $\left(n_{2}, d\right)$ are unknown and rest of them are constants. Theoretically, the $\left(n_{2}, d\right)$ can be determined if corresponding $(\psi, \Delta)$ are calculated by using Equation (14).

However, given that it is hard to derive analytical solutions, a range of $\left(n_{2}, d\right)$ will be taken in order to find appropriate sets of values.

1) Suppose there is no light absorption

When there is no absorption, the idea is simple by using exhaustion method, as illustrated by Figure 7: Make $n_{2}$ varies from 0.01 to 5.01 with step length of 0.01 . and make d to vary from $1 \mathrm{~nm}$ to $1504 \mathrm{~nm}$ with step length $3 \mathrm{~nm}$. When $R_{p} / R_{s}$, which is derived by $\left(d, n_{2}\right)$ is in a certain range of the $\tan (\psi) \exp (i \Delta)$, which is derived from measured the value, then, output the $\left(d, n_{2}\right)$ as a set of solution. Known that most of value will be out of range, we divide the approximation into two steps: first step is selecting the values in low accuracy, narrowing the range, the second step has smaller scope of approximation. This method can reduce the searching time due to the elimination of searching out of the possible range while increasing the accuracy.

It is noticeable that the result of thickness can be a periodic number. The period of thickness can be calculated by equation $d=\lambda / 2 \sqrt{n_{2}^{2}-n_{1}^{2} \sin ^{2} \varphi_{1}}$ [2]. If the thickness is beyond one period, we need the data set from another incidence angle. According to the estimation, the thickness of film in this experiment is within one period, so another set of data is not needed. The final result of estimation is $d=42 \mathrm{~nm}$ and $n_{2}=1.332$.

2) Counting light absorption

When counting the light absorption of the film, we define the extinction coefficient of the film as $\mathrm{K}$ and refractive index as $\mathrm{N}$, so $n_{2}=N-k i$. When finding the optimized combination of $(N, k, d)$ with exhaustion method requires extended period of searching.

Particle Swarm Optimization, PSO is an optimization algorithm inspired by bird flocks searching for food. Every imagined particle is searching for optimal position in a multi-dimensional space. Every particle has its velocity and position. By continuous refreshing position and velocity, particles finally reach the optimal point [3].

There are a number of steps taken by the algorithm. It is also illustrated in Figure 8.

\subsection{Initiation}

Define the fitness function as $X^{2}=\tan (\psi) \mathrm{e}^{-2 i \delta}-R_{p} / R_{s}$. Define the Maximum iteration number $\mathrm{M}$ as 100 , number of particle $\mathrm{N}$ as 50 . Set the number of dimensions as 3 . We generated the initial position and velocity of particles randomly.

\subsection{Compare the Fitness}

Compare the value of fitness function of the current position and previous position. Let individual particle find the individual optimization point, then generating the group optimization point. Compare the value of fitness function at the current optimization point with the historical group optimization point, and then refresh the position and velocity.

\subsection{Refresh of the Velocity and Position}

The refresh of the velocity and position is according to the following expression (16) and (17) [4]

$$
V_{i d}=\omega V_{i d}+C_{1} \operatorname{random}(0,1)\left(P_{i d}-X_{i d}\right)+C_{2} \operatorname{random}(0,1)\left(P_{g d}-X_{i d}\right)
$$




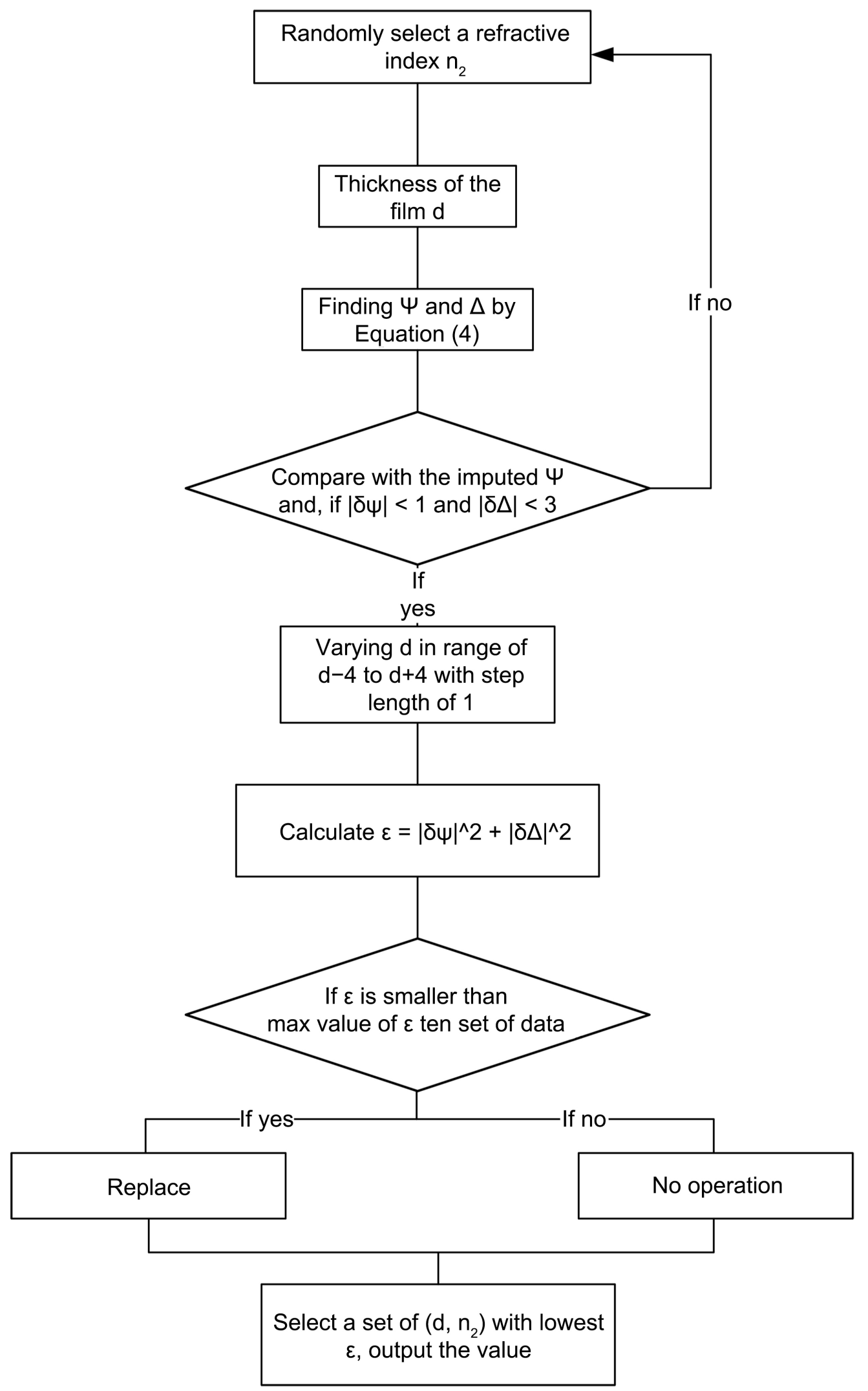

Figure 7. Programming procedure when suppose there is no light absorption cause by the film coated. 


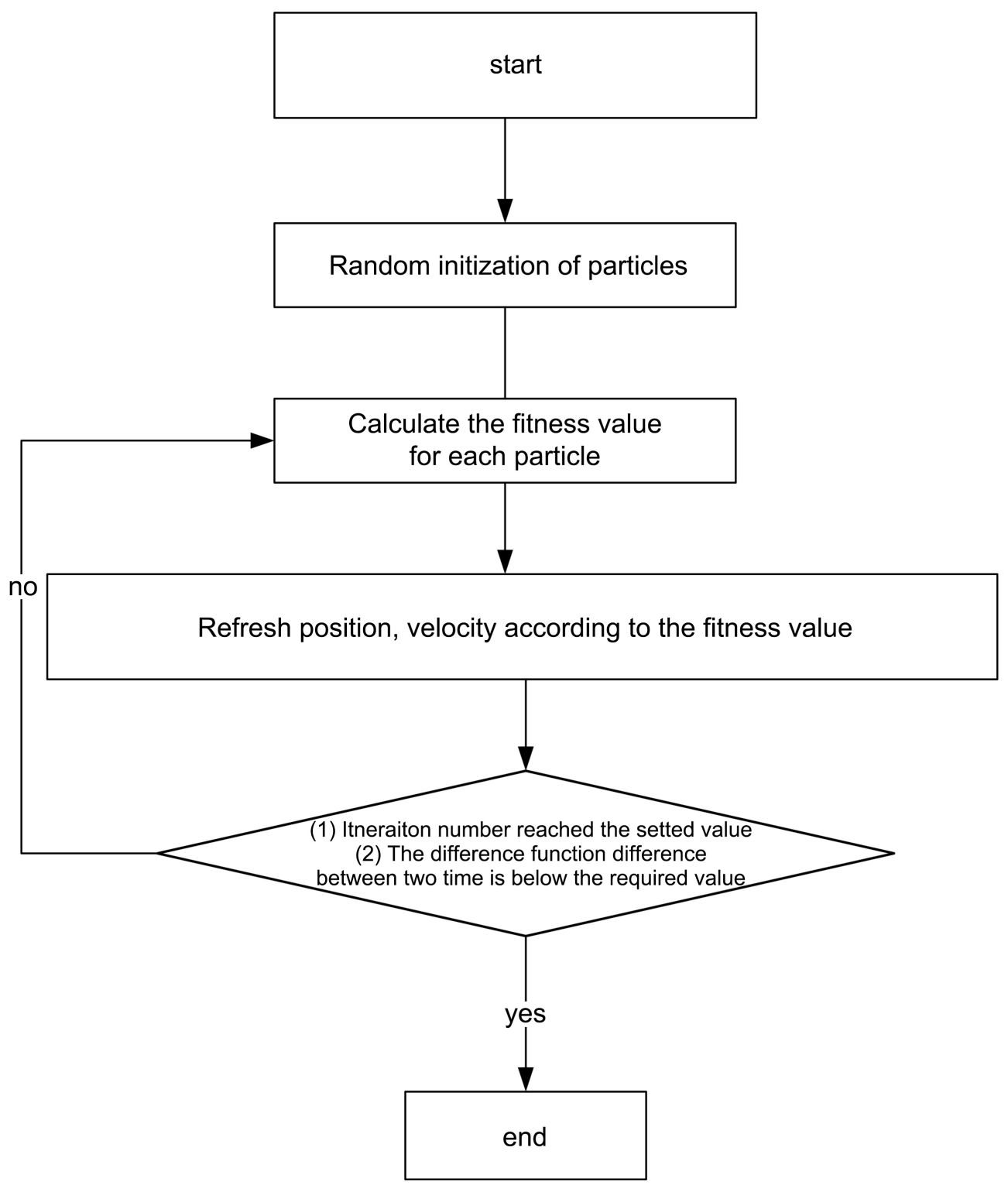

Figure 8. The programming procedure of PSO.

$$
X_{i d}=X_{i d}+V_{i d}
$$

In the expressions, $\omega$ is the inertia coefficient, which is non-negative. The greater the number, the greater the ability of particle to search in large scope. $C_{1}$ and $C_{2}$ are learning coefficient. They are empirical value from 0 to 4 . Here, $C_{1}$ and $C_{2}$ are 1.5 and 2.5. $i$ is representing which particle, and $\mathrm{d}$ is representing the dimension. $V, X, P$ is referring to velocity, fitness value, and position. The refreshment is according to the "experience" attained by other particles.

\subsection{End Condition}

1) Iteration number reached the preset value.

2) The difference function difference between two times is below the required value.

In order to prevent the particles, end at regional optimization point instead of general optimization point, we confine the particle in a range in each dimension in initial condition: $\mathrm{N}$ between 1 to 2 , $\mathrm{k}$ between 0 to 10 , and the thickness is between $0-100 \mathrm{~nm}$. The final result of optimization is $\mathrm{N}=1.442 \mathrm{k}=$ 
$0.543 \mathrm{~d}=40.033 \mathrm{~nm}$. Close to the parameter provided by manufacture which has $\mathrm{N}$ equals to 1.44 and $\mathrm{d}$ equals $41.01 \mathrm{~nm}$.

\section{DISCUSSION AND CONCLUSION}

By using a novel set up to measure the phase difference between $\mathrm{S}$ and $\mathrm{P}$ component of light and adopting conventional methods in calculating thickness and refractive index of film in Ellipsometry which are PSO and exhaustion method, we successfully achieved the result we want. Compared to ellipsometry, since the newly adopted method for measuring phase difference, such method can be applied to the condition when the light is incidence from optically dense material to optically less dense material.

\section{ACKNOWLEDGEMENTS}

I would like to acknowledge the contributions of my advisor Mrs. Yuan, who provided instruction on the format of essay writing during her break time. With her help, I can present my ideas and finding in a well-formatted and logical paper. I would like to extend my special thanks to Shanghai Pinghe School for provision of laboratory.

\section{CONFLICTS OF INTEREST}

The authors declare no conflicts of interest regarding the publication of this paper.

\section{REFERENCES}

1. Chaturvedi, S., Pragya, P. and Verma, H.K. (2015) Comparative Analysis of Particle Swarm Optimization, Genetic Algorithm and Krill Herd Algorithm. 2015 International Conference on Computer, Communication and Control(IC4), Indore, 10-12 September 2015, 1-7. https://doi.org/10.1109/IC4.2015.7375552

2. Ghimire, K., Cimaroli, A., Hong, F., Shi, T., Podraza, N. and Yan, Y. (2015) Spectroscopic Ellipsometry Studies of CH3NH3PbX3 Thin Films and Their Growth Evolution. 2015 IEEE 42nd Photovoltaic Specialist Conference (PVSC), New Orleans, LA, 14-19 June 2015, 1-5. https://doi.org/10.1109/PVSC.2015.7356387

3. Oostra, B. (1970) A Method for Measuring Magnetic Fields in Sunspots Using Zeeman-Broadened Absorption Lines. American Association of Physics Teachers, American Association of Physics Teachers AAPT, 1 January 1970.

4. Yu, P., et al. (2011) Two Diode Lasers Simultaneously Optically Locked by Orthogonal Polarizations of a Monolithic Confocal Cavity. Chinese Physics Letters, 28, Article ID: 104208.

https://doi.org/10.1088/0256-307X/28/10/104208 